\title{
Diagnostic Markers for Early Detection of Neonatal Sepsis
}

\author{
Lara R. Shoukry ${ }^{1}$, Ahmed N. Mohamed ${ }^{1}$, Alzahraa E. A. Sharaf ${ }^{1}$, Osama B. S. Osman ${ }^{1}$ \\ ${ }^{I}$ Department of Clinical And Chemical Pathology, Faculty of Medicine, Sohag University
}

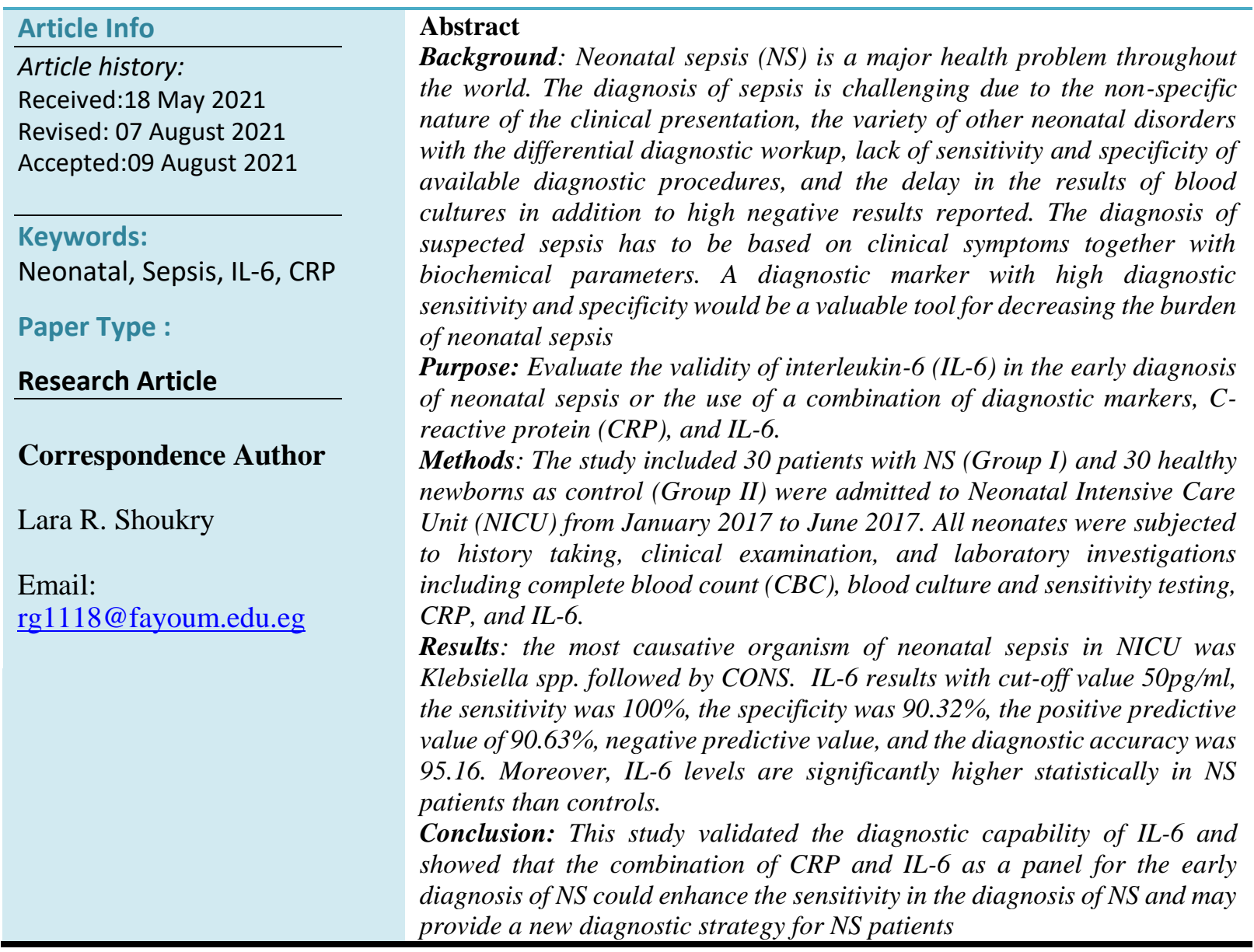

\section{Introduction}

Neonatal sepsis is the commonest cause of neonatal mortality; it is responsible for about $30-50 \%$ of the total neonatal deaths in developing countries. It is estimated that up to $20 \%$ of neonates develop sepsis and approximately $1 \%$ die of sepsis-related causes (Ahmad et al., 2016). The reasons are mainly related to the combination of the neonatal reduced immune defense and the complex interactions between the infecting microorganisms and host response (Shane et al., 2017). Unnecessary and increased antibiotic consumption leads to a higher incidence of the side-effects due to their use, increased resistance to antibiotics, a long hospitalization and increased health costs. Therefore, using fast diagnostic methods including laboratory markers could be beneficial for the diagnosis of neonatal sepsis (Bindlish et al., 2015). 
There is no single reliable test for the early definite diagnosis of neonatal sepsis, and therefore, there is a continuing search for a new infection marker to identify the most suitable serum marker with reasonable sensitivity and specificity in the diagnosis of neonatal sepsis (Kheir et al., 2013).

C-reactive protein (CRP) is the most extensively studied acute-phase reactant so far, and despite the ongoing rise (and fall) of new infection markers, its wide availability and its simple, fast, and cost-effective determination make it one of the preferred indices in many neonatal intensive care units (NICUs) (Dulay et al., 2015). The principal ligand to CRP with the highest binding affinity is phosphocholin, which is found in lipopolysaccharide and cell walls of many bacteria and microorganisms as well as in the outer leaflet of most biological membranes (Ngwa \& Agrawal, 2019).

Interleuin-6 (IL-6) is the most frequently studied cytokine in the diagnosis of bacterial infections in the neonates. As in most cases of neonatal sepsis, IL- 6 increases rapidly after exposure to bacterial products, several hours before the increase in the concentration of CRP, and decreases within 24 hours to undetectable levels even though the infection persists (Chirico \& Loda, 2011). The concentration of IL-6 in preterm and term infants does not seem to be influenced by gestational age or maternal cytokine concentration (Sherwin et al., 2008).

\section{Subjects and Methods}

This is a case-control study that was conducted in the period from "January 2017 to June 2017" in NICU, Sohag University Hospital, Egypt. This study included a total number of 60 newborns who were classified into two groups each of 30 neonates; Group I included the newborns are diagnosed by a pediatric physician according to clinical symptoms, complete blood picture, positive CRP and positive blood culture and group II that included 30 healthy neonates.

All newborns between 0 and 28 days admitted to NICU were included in the study. The cases with the following conditions were excluded; age greater than 28 days, newborns under antibiotic treatment before enrollment and newborns with congenital anomalies. After approval from the local Ethical Scientific Committee of Sohag Faculty of Medicine and obtaining informed written consent from the guardians of the neonates, all cases were subjected to complete history taking and through full general examination. The following laboratory investigations were done for all the cases (at time of admission and before starting antibiotics treatment) including complete blood count (CBC), blood culture and sensitivity testing, serum C-reactive protein (CRP) level and serum interleukin-6 (IL-6) level.

\section{Technique}

Five $\mathrm{ml}$ of blood samples were collected from the newborns under complete aseptic precautions in the NICU, $2 \mathrm{ml}$ in EDTA-vacutainer for complete blood count, $1 \mathrm{ml}$ for conventional blood culture and sensitivity testing, and the remaining $2 \mathrm{ml}$ were collected in plain tubes to allow samples to clot for 2 hours at room temperature before centrifugation for 15 minutes at $1000 \times \mathrm{g}$. After that, pipetted without agitation to be used for CRP assay and the 
remaining serum sample was collected and divided into aliquots and stored at $-20^{\circ} \mathrm{C}$ for detection of IL-6 level by enzyme-linked immunosorbent assay (ELISA).

Complete blood count was performed on an automatic cell counter (ABX Pentra 60) manufactured by (HORIBA ABX SAS). Serum CRP level was measured by the semiquantitative latex agglutination test (BioMed. CRP kits; Catalog No. CRP301100; supplied by Egy-Chem Diagnostics) according to the manufacture's guideline. IL-6 in serum was measured by Human IL-6 (Interleukin 6) ELISA Kit supplied by Elabscience Biotechnology Co. (Catalog No: E-EL-H0102/ 96T). For blood culture test, blood was placed in the BACTECTM 9050 blood culture instrument within two hours of collection; at $37^{\circ} \mathrm{C}$, inspected daily for 3 days for the presence of visible microbial growth or not to label it sterile. Subcultures were done in positive cases to identify the causative organism according to the standard methods. Thereafter, antibiotic susceptibility testing was performed on Mueller Hinton agar (Oxoid) by Kirby-Bauer disk diffusion method.

\section{Statistical analysis}

Data entered and analyzed using Microsoft Excel software. Data were then imported into Statistical Package for the Social Sciences (SPSS 24.0, IBM/SPSS Inc., Chicago, IL) software for analysis. Baseline characteristics of the study population were presented as frequencies and percentages (\%) or mean values and standard deviations (SD) and (Range). For comparison of data, the Chi-Square test (or Fisher's exact test) was used to compare two independent groups of qualitative data. For quantitative data, independent-Samples t-test and Mann-Whitney $U$ test were used to compare two groups of parametric and non-parametric quantitative data respectively. $\mathrm{P}$ values $<0.05$ are considered significant. Sensitivity, specificity, positive and negative predictive values and diagnostic accuracy were calculated in accordance with standard methods.

\section{Results and Discussion}

As shown in table (1), there was no statistically significant difference in the sex distribution and age between the two study groups. twenty patients $(66.7 \%)$ of (NS group) were males while ten $(33.3 \%)$ of patients were females. In (control group), 17 (56.7\%) were males and $13(43.3 \%)$ were females. The male: female ratio was 2:1. According to the gestational age, 17 cases $(56.7 \%)$ in the study group were preterm infants ( $<37$ weeks of gestational age), in opposite to 10 neonates $(33.3 \%)$ in the control group. There was no statistically significant difference between the study and control groups regarding the gestational age $(\mathrm{P}>0.05)$. in the sepsis group, 23 cases $(76.7 \%)$ were considered as EOS as sepsis starts in the first 7 days of life while 7 cases $(22.3 \%)$ were considered as LOS as sepsis starts after that. The weight at admission in the study group was with range $(1560 \mathrm{~g}-3940 \mathrm{~g})$ and mean of $(2723.17 \pm 687.98)$ while in the control group, the range was (1600g-3900g), and the mean was (2632.0 \pm 646.44$)$. 
Table 1: Basic data in study and control groups

\begin{tabular}{|c|c|c|c|c|c|}
\hline \multirow[t]{2}{*}{ Variables } & \multicolumn{2}{|c|}{$\begin{array}{l}\text { Group I } \\
\text { NS } \\
(n=30)\end{array}$} & \multicolumn{2}{|c|}{$\begin{array}{l}\text { Group II } \\
\text { Control } \\
(n=30)\end{array}$} & \multirow[t]{2}{*}{ P. value } \\
\hline & No. & $\%$ & No. & $\%$ & \\
\hline \multicolumn{6}{|l|}{ Sex } \\
\hline Male & 20 & 66.7 & 17 & 56.7 & \multirow[t]{2}{*}{0.426} \\
\hline Female & 10 & 33.3 & 13 & 43.3 & \\
\hline \multicolumn{6}{|l|}{ Age } \\
\hline Up to 7 days & 23 & 76.7 & 22 & 73.3 & \multirow[t]{2}{*}{0.573} \\
\hline From 8 to 30 days & 7 & 22.3 & 8 & 26.7 & \\
\hline \multicolumn{6}{|l|}{ gestational age } \\
\hline Pre term $<37$ weeks & 17 & 56.7 & 10 & 33.3 & \multirow[t]{2}{*}{0.069} \\
\hline Full term $>37$ weeks & 13 & 43.3 & 20 & 66.7 & \\
\hline Weight (gram) & \multicolumn{2}{|c|}{$2723.17 \pm 687.98$} & \multicolumn{2}{|c|}{$2632.0 \pm 646.44$} & 0.599 \\
\hline \multicolumn{6}{|l|}{ Onset of sepsis } \\
\hline Early(birth-7) days & 23 & 76.7 & & & \\
\hline Late (8-30) days & 7 & 23.3 & & & \\
\hline
\end{tabular}

Source: Authors

Table (2) shows that there was no statistically significant difference in the RBCs count or platelets count between the cases in the two study groups. Leukopenia was present in 10 patients in the study group while it was present in 3 neonates in the control group. On the other hand, leukocytosis was presented in 7 patients in the study group and one neonate in the control group. As among 29 blood culture-positive cases, abnormal WBCs count was detected in 6 cases $(13.8 \%)$ while among 31 blood culture-negative cases, abnormal WBCs count was detected in 2 cases $(6.5 \%)$. There was a high statistically significant difference between study and control groups regarding as $40 \%$ of cases show abnormal TLC in opposite to $13.3 \%$ of neonates in the control group.

Table 2: Complete blood count in the two studied groups

\begin{tabular}{|c|c|c|c|c|c|}
\hline \multirow[t]{2}{*}{ variables } & \multicolumn{2}{|c|}{$\begin{array}{l}\text { Group I } \\
\text { NS } \\
(n=30)\end{array}$} & \multicolumn{2}{|c|}{$\begin{array}{l}\text { Group II } \\
\text { Control } \\
(n=30)\end{array}$} & \multirow[t]{2}{*}{ *P. value } \\
\hline & No. & $\%$ & No. & $\%$ & \\
\hline RBCs $\times 10^{3} / \mathrm{cmm}$ & \multicolumn{2}{|c|}{$3909.33 \pm 983.93$} & \multicolumn{2}{|c|}{$4187.67 \pm 721.95$} & 0.599 \\
\hline \multicolumn{6}{|l|}{ WBCs count } \\
\hline $\begin{array}{l}\text { Normal } \\
5000-20000 \\
\text { cell/cmm }\end{array}$ & 12 & 40.0 & 26 & 86.7 & \multirow[t]{2}{*}{$<0.001$} \\
\hline $\begin{array}{l}\text { Abnormal } \\
<5000 \& \&>20000 \\
\text { cell/cmm }\end{array}$ & 18 & 60.0 & 4 & 13.3 & \\
\hline Platelets count & \multicolumn{2}{|c|}{$295633.33 \pm 129724.24$} & \multicolumn{2}{|c|}{$326266.67 \pm 104197.40$} & 0.317 \\
\hline
\end{tabular}

Copyright (C 2020-2021, Journal of Scientific Research in Medical and Biological Sciences (JSRMBS), Under the license CC BY- 4.0 
Table (3) shows the blood culture result distribution in study and control groups. There was a high statistically significant difference between study and control groups ( $\mathrm{p}$. value < 0.01). The relative frequency of the causative microorganisms of the studied neonates according to blood culture is also presented, showing Klebsiella pneumonia as the most frequent bacteria. There were $29(49,3 \%)$ patients had positive blood cultures, and 31(51.7\%) neonates had negative blood cultures.

The higher sensitivity was for vancomycin. As vancomycin and amoxyclav exhibited good activity against both, Staph aureus and CONS. The gram-positive bacteria were resistant to Penicillin (Data not shown).

Table 3: The blood culture results between study \& control groups

\begin{tabular}{|c|c|c|c|c|c|}
\hline \multirow[t]{2}{*}{ BLOOD CULTURE } & \multicolumn{2}{|c|}{ Case $(n=30)$} & \multicolumn{2}{|c|}{ Control $(n=30)$} & \multirow[t]{2}{*}{ P. value } \\
\hline & No. & $\%$ & No. & $\%$ & \\
\hline Negative (No growth) & 3 & 10.0 & 28 & 93.3 & \multirow[t]{6}{*}{$<0.001$} \\
\hline Candida & 1 & 3.3 & 1 & 3.3 & \\
\hline CONS & 7 & 23.3 & 0 & 0.0 & \\
\hline E.COLI & 5 & 16.7 & 1 & 3.3 & \\
\hline KLEBSIELLA SPP. & 8 & 26.7 & 0 & 0.0 & \\
\hline STAPH AUREUS & 6 & 20.0 & 0 & 0.0 & \\
\hline
\end{tabular}

Source: Authors

Table (4) shows that the mean value of CRP of (21.4 \pm 14.92$)$ and the range was 6-48 in the study group (CRP cut-off level $=6 \mathrm{mg} / \mathrm{dl}$. the IL-6 level in the study group with a mean value of (286.84 \pm 100.79$)$ and in the control group with a mean value of $(37.24 \pm 55.41)$. IL-6 showed a high statistically significant difference in the study group as compared to the control group (p. value $<0.001$ ).

Table 4: CRP and Serum IL-6 in study and control groups

\begin{tabular}{|l|l|l|l|l|l|}
\hline \multirow{2}{*}{} & \multicolumn{2}{|l|}{ Case $(\mathrm{n}=30)$} & \multicolumn{2}{l|}{ Control $(\mathrm{n}=30)$} & \multirow{2}{*}{ P. value } \\
\cline { 2 - 5 } & No. & \% & No. & \\
\hline $\begin{array}{l}\text { CRP } \\
(\text { Mean } \pm \text { SD) }\end{array}$ & $\begin{array}{l}21.4 \pm 14.92 \\
(6-48)\end{array}$ & \multicolumn{2}{|l}{} & \\
\hline IL-6 & & & & & \\
\hline $\begin{array}{l}\text { Negative } \\
<50 \mathrm{pg} / \mathrm{ml}\end{array}$ & 0 & 0.0 & 28 & 93.3 & $<0.001$ \\
\hline $\begin{array}{l}\text { Positive } \\
>50 \mathrm{pg} / \mathrm{ml}\end{array}$ & 30 & 100.0 & 2 & 6.7 & \\
\hline Mean \pm SD & $286.84 \pm 100.79$ & $37.24 \pm 55.41$ & \\
\hline
\end{tabular}

Source: Authors

As shown in table (5), the hematological indices (TLC \& Thrombocytopenia) had uniformly poor sensitivity but good specificity and PPV, this study further showed the best diagnostic accuracy for culture-proven sepsis was achieved by the combination of IL- 6 \& CRP as they identify all infected infants followed by IL-6 alone or CRP alone. 
Table 5: Comparative analysis of tests used in the study

\begin{tabular}{|l|l|l|l|l|l|}
\hline & Sensitivity & Specificity & PPV & NPV & Accuracy \\
\hline TLC & 58.62 & 83.87 & 77.27 & 68.42 & 71.25 \\
\hline Thrombocytopenia & 13.8 & 100 & 100 & 54.5 & 56.9 \\
\hline CRP & 93.1 & 90.32 & 90 & 93.3 & 91.71 \\
\hline IL6 & 100 & 90.32 & 90.63 & 100 & 95.16 \\
\hline CRP+IL6 & 100 & 90.32 & 90 & 100 & 95.16 \\
\hline
\end{tabular}

Source: Authors

In this study, we investigated the IL-6 level of patients with NS with respect to NS free controls who are apparently healthy and its diagnostic value for NS with CRP. To achieve this goal, we selected 30 patients with NS, 30 apparently healthy newborns, virtually symptoms free and CRP level $<6 \mathrm{mg} / \mathrm{dl}$. In the NS group, there were 20 males (66.7\%) and 10 females $(33.3 \%)$ with ratio of $2: 1$. This is in agreement with the study by Jeyaganguli $D$ et al. (2018) and Vinay et al. (2015).

Among the infected newborns, the predominance of the males was due to the factors regulating the synthesis of globulin are situated on the $\mathrm{X}$ chromosome. Male has only one $\mathrm{X}$ chromosome; he is less immunologically protected than the females (Bhale et al., 2016). In the study group, the rate of prematurity was high $(56.7 \%)$ which is far more than the incidence of prematurity in the general population which may indicate predilection of suspected sepsis more in case of the premature neonate. These results are in agreement with Labib et al. (2013). Preterm and very low birth weight babies are more susceptible to infection due to low levels of IgG and immature lower defense mechanisms and invasive life support systems make the premature neonate particularly susceptible to overwhelming infection.

Early-onset sepsis was seen in (76.7\%) cases while the remaining (23.3\%) were of lateonset sepsis. These findings are similar to a study done by Gandhi et al. (2013). Also, Chugh et al. (1988) in which they have reported EOS cases to be more in number than LOS, they reported K. Pneumoniae as the commonest isolate in both EOS and LOS.

The vast majority of studies in that literature conclude that hematological indices have low sensitivity and specificity in identifying EOS, being more useful in identifying newborns with low risk for sepsis. Six to 12 hours are necessary before the effect of the inflammatory response on the number and ratio of immature and mature neutrophil occurs. An extensive review performed by Da Silva et al. (1995) that found a large range of sensitivities - 17-90\% and specificities - 31-100\% - for leukocytes and leukocyte indices in EOS screening, explained the results by laboratory experience variances, different postnatal age of the patients, and influence of non-infectious factors affecting neutrophils parameters and concluded that serial measurements of the leukocyte indices may increase the accuracy of EOS diagnosis (Da Silva et al., 1995).

Total leukocyte count (TLC) is of little clinical use in the diagnosis of neonatal infection because of the wide variation in values. In this study, TLC was not abnormal in all cases $(60 \%)$ in opposite to $13.3 \%$ of the control group due to early collection, previous low level, and the 
high percentage of prematurity in the study group. The sensitivity of TLC was $58.62 \%$, specificity of $83.87 \%$, with PPV $77.27 \%$, and NPV $68.42 \%$ which were consistent with the study results by Vinay et al. (2015).

This study further showed that sensitivity and specificity of TLC are lower as compared to that of CRP \& IL-6; therefore, these indices render it less valuable for screening or detection purposes. Neutropenia can be a better diagnostic marker of neonatal sepsis than neutrophilia because the marrow reserve of leukocytes in a newborn is relatively smaller compared with older children and adults and it has greater specificity as few conditions (maternal pregnancyinduced hypertension, asphyxia, and heamolytic disease) other than sepsis depress the neutrophil count of neonates (Polin, 2012).

In general, the abnormal leukocyte counts, such as leukopenia and neutropenia tend to have high specificity as our study results while their sensitivity is slightly lower. These limitations in sensitivity and specificity due to the relatively long period necessary to become positive, the significant influence of non-specific factors, and the interobserver variations, affect its role in early diagnosis of NS (Emami et al., 2016; Shah et al., 2014). Neonates with sepsis develop thrombocytopenia, possibly because of disseminated intravascular coagulation (DIC) and the damaging effects of endotoxin on platelets especially presented in LOS cases. In this study, we found thrombocytopenia in $8.33 \%$ of cases with a sensitivity of $13.8 \%$, specificity $100 \%$, PPV $100 \%$, and NPV $54.5 \%$. These results were consistent with the results by Ahmad et al. (2016).

Despite the frequency of low platelet counts in infected infants, they are an insensitive and late indicator of sepsis. Moreover, platelet counts are not useful to follow clinical response to antimicrobial agents, because they often remain depressed for days to weeks after a sepsis episode which is in agreement with our study. The low percentage of thrombocytopenia $8.33 \%$ in the study group may be due to the low percentage of LOS cases in the study group. Also, there is no statistically significant difference between the study and control groups regarding platelets count which means that their diagnostic and screening role is weak.

The presence of leukopenia and thrombocytopenia are poor screening tools for neonatal sepsis. However, when present, they are strong pointers to the presence of sepsis. These hematological abnormalities are defining criteria for systemic inflammatory response syndrome (SIRS) and severe sepsis in early infancy. However, every infection does not progress to SIRS; so many cases may be missed in the early stages and thus resulting in lower sensitivities of hematological parameters when used for sepsis screening. Putatively, the occurrence of leucopenia and thrombocytopenia represent a relatively advanced stage of infection (Ahmad et al., 2016).

Anemia is not a constant feature in neonate with positive blood culture, moreover, some neonates with blood film showing a mild degree of anemia that may be physiological or attributable to prematurity (Poornima, 2017) which is in agreement with our results in which there is no statistically significant difference between study and control groups regarding RBCs count. The hematological parameters studied have not shown high sensitivity or specificity in many other studies as well and the results have been varied. The variation may be partly 
explained by differences in case definition, in the blood sampling time, the severity of infection, the age of the neonates, culture methods used and the diagnostic criteria followed.

In our study, 29 babies (48.3\%) were proven to have neonatal sepsis based on positive blood culture results in agreement with Abdelmoneim et al. (2013). This is different from a study done by Mandour et al. (2014) also found a percentage of 78,26\% for the sensitivity of culture. The total number of pathogenic isolates was 29 , out of which 2 isolates were of candida albicans and the rest 27 were bacterial isolates. Gram-negative bacteria (48.28\%) were more frequently isolated than gram-positive bacteria (44.83\%) with K. spp. accounting for a maximum of (27.59\%) of all bacterial isolates. CONS were the second most commonly isolated pathogen in agreement with the study by Hisamuddin E et al. (2015).

Overall, the isolation of gram-negative bacteria was higher than gram-positive bacteria. These results were consistent with the finding of Aftab and Iqbal (2006). Most of the studies carried out in developing countries have shown K. pneumoniae as the most implicated gramnegative bacteria for NS as in Mondal et al. (1991). But Süner et al. (2014) found that the distributions of the microorganisms isolated from blood culture were as follow: Staphylococci at $32.8 \%$, Enterococcus spp. at 9.3\%, Acinetobacter spp. at $18.7 \%$, P. aeruginosa at $4.6 \%$, Klebsiella spp. at $4.6 \%$.

The antibiotic susceptibility pattern of all the isolated organisms also studied and showed that the gram-positive bacteria were resistant to penicillin. Vancomycin and amoxiclav exhibited good activity against both, Staph aureus and CONS which is similar to a study conducted by kayange et al. (2010). Although the gold standard method for diagnosis of neonatal sepsis is the isolation of microorganism from blood, it is a time-consuming procedure usually takes more than three days for complete result and also requires well-equipped laboratory and trained personnel for better results. Hence the alternative fast diagnostic test of serological markers enabling earlier detection of neonatal sepsis might be beneficial (Jeyaganguli et al., 2018).

The ideal diagnostic marker should have a very high sensitivity and negative predictive value preferably reaching $100 \%$. However, the lack of reliable clinical signs often results in the injudicious use of antibiotics. So, an ideal diagnostic marker should also have high specificity and a good positive predictive value preferably better than $85 \%$ to minimize unnecessary use of antibiotics in false-positive cases (Emami et al., 2016).

According to Ahmad et al. (2016), when a severe bacterial infection is suspected, the high sensitivity of a test might be more important than high specificity to avoid making a falsenegative diagnosis. However, for diseases that are not life-threatening, a high specificity is preferable to avoid unnecessary hospital admissions and the unnecessary use of antibiotics (Ahmad et al., 2016).

In our study, the mean CRP level was significantly higher in patients $(21.4 \pm 14.92)$ than controls in agreement with Labib et al. (2013). So, this parameter was affected in the suspected sepsis group and differentiates them from healthy neonates and progressively increases through the course of illness reaching significantly higher levels when sepsis is proven and our data confirmed the previous study by Ayazi et al. (2014). 
For the diagnosis of early-onset sepsis in clinical practice, the sensitivity is more important compared to the specificity, as the consequences of unnecessarily treating an uninfected infant bear fewer complications than not treating an infected child (Hofer et al., 2011).

The sensitivity of CRP is known to be the lowest during the early stages of infection. For a single CRP determination at the time of initial evaluation as well as for determinations from cord blood, the CRP diagnostic accuracy varies widely within an unacceptable range of sensitivity to guide therapy either by reliably diagnosing or excluding bacterial infection (Hofer et al., 2013). Meem et al. (2011) reported that the optimum sensitivity and specificity for CRP were obtained during the window of 24-48 $\mathrm{h}$ after the onset of symptoms. Benitz et al. (1998) also suggested that serial measurements of CRP over a period of 2-3 days after onset clinical symptom, using varying cutoff values, improved the diagnostic performance of CRP.

The specificity and positive predictive value of CRP in this study was $90 \%$ in agreement with Birju Shaha and James Padbury (2014) as said that specificity and positive predictive value of CRP range from 93-100\% (Shah \& Padbury, 2014). Reliable reference values of CRP are crucial for obtaining adequate diagnostic accuracy. Upper limits for CRP during the first days of life have mainly been established from uninfected but symptomatic neonates. The studies assessing upper limits in healthy neonates were mostly based on rather small sample sizes or did not take into account their postnatal age. The cut-off value is an appropriate threshold above which results can be considered abnormal. These values reported in the literature range from 1.5 to $20 \mathrm{mg} / \mathrm{L}$ with wide-ranging sensitivities and specificities (Chiesa et al., 2004).

There are also several studies that have used different cutoff values for CRP ranging from 4.8 to $70 \mathrm{mg} / \mathrm{l}$ and have reported that the sensitivity of CRP for identifying neonatal infection ranges from $63 \%$ to $95 \%$, and specificity ranges from $40 \%$ to $97 \%$ (Celik et al., 2010). IL-6 is an important cytokine of the early response to infection. Previous studies have shown IL-6 to be a useful marker of early infection in the newborn. In our study, the serum IL-6 levels were significantly increased in newborns with sepsis compared with control in agreement with the study by Marwa El-Sonbaty et al. (2016). Another study by Magudumana MO et al. (2000) showed that septic preterm newborns had significantly elevated IL-6 levels at the onset of sepsis as compared to the recovery period and the controls. Also, he reported that there is no benefit in the serial determination of IL- 6 in the diagnosis of neonatal sepsis.

Our results showed that IL-6 shows $100 \%$ sensitivity at $50 \mathrm{pg} / \mathrm{ml}$ cutoff value. The cutoff values obtained by other investigators who measured IL- 6 in neonatal sepsis ranged between 3.6 and $500 \mathrm{pg} / \mathrm{ml}$ with mean cutoff value $=76.49 \mathrm{pg} / \mathrm{ml}$ and median $30 \mathrm{pg} / \mathrm{ml}$. The mean sensitivity was $77.87 \%$ and specificity was $78.61 \%$, at $76.49 \mathrm{pg} / \mathrm{ml}$ cutoff value (Meem et al., 2011).

Noor et al. (2008) found that the validity of the IL-6 test in comparison to blood culture cases was $100 \%$ in agreement with ours. Although IL-6 is considered one of the early markers in neonatal sepsis, some factors affect its sensitivity and specificity to be widely used in neonatal diagnosis. Its concentration increases sharply after exposure to bacterial infection and it even precedes the increase in CRP but it has a very short half-life, and its concentration falls dramatically with treatment and becomes undetectable within $24 \mathrm{~h}$. In addition, it was reported 
that the level of IL-6 shows natural fluctuations immediately after the postnatal period and its serum level is affected by gestational age and perinatal complications other than infection (ElSonbaty 2016).

Earlier studies have suggested that IL-6 derived cutoff levels of 20-50 pg $\mathrm{ml}$ in the peripheral blood of neonates seem to be the most promising marker for neonatal EOS Bhandari et al. (2008) in agreement with our study results. However, reports in that literature on the use of CRP, and IL-6 as early markers of neonatal sepsis are contradictory. Variations in study design, the definition of neonatal sepsis, sample size, postnatal age, gestational age, risk factors, inclusion criteria of patients, cutoff points of the markers, test methodology, data analysis, and reporting of results lead to difficulties in comparing studies. Thus, it is often difficult to formulate a definitive opinion on the clinical usefulness of infection markers from the published reports (Ucar et al., 2008).

In this study, both sensitivity and specificity of CRP are near to these of IL-6 but both measurements at the same time ensure the diagnosis of NS as the diagnostic accuracy of both (95.61\%) is higher than that of CRP alone. In a study done by Doellner et al. (2001) in Norway, 6 inflammatory mediators including CRP and IL-6 were compared as early diagnostic tests for neonatal sepsis and the possible benefit of combining parameters was studied. CRP has a high diagnostic performance and the diagnostic accuracy was further improved by combining CRP and IL-6 which is in agreement with our study.

While evaluating the findings obtained from this study, methodological limitations such as the small sample size and the absence of follow-up for septic and clinically suspected septic neonates must be taken into account. However, findings that were obtained from this study were strengthened by the following factors: the presence of positive blood cultures in all of the septic neonates; taking newborns at risk of neonatal sepsis development and taking healthy newborns as a control group; the exclusion of neonates who were either delivered by a mother that was using antibiotics or had used antibiotics before.

\section{Conclusion}

The study demonstrated that serum IL-6 levels were comparable to those of CRP in the NS group of patients, so serum IL-6 may serve as a good diagnostic biomarker for the diagnosis of NS especially in high-risk patients, IL-6 alone or in combination with CRP significantly improve the diagnostic accuracy of NS with a sensitivity, specificity, and accuracy higher than that of CRP alone.

\section{Conflict of Interest}

Authors declare no conflicts of interest.

\section{References}

Ahmad, I., Ahmed, A., Abrar, S. S., Lone, M. R., Khanday, M. R., Bhat, J. I., ... \& Ahmed, S. M. (2016). Changing role of neonatal sepsis markers in the era of BacT/Alert: is there 
a need to devise a new septic screen. International Journal of Contemporary Pediatrics, 3(1), 107.

Aftab, R. U. S. H. D. A., \& Iqbal, I. (2006). Bacteriological agents of neonatal sepsis in NICU at Nishtar Hospital Multan. Journal of the College of Physicians and Surgeons-pakistan: JCPSP, 16(3), 216-219.

Bindlish, S., Goyal, G., \& Panag, K. (2015). Role of C-reactive protein (CRP) as a screening tool in early diagnosis of neonatal septicemia. Int. J. Biomed. Res, 6(02), 125-130.

Bhandari, V., Wang, C., Rinder, C., \& Rinder, H. (2008). Hematologic profile of sepsis in neonates: neutrophil CD64 as a diagnostic marker. Pediatrics, 121(1), 129-134

Bhale, C. P., Kale, A. V., Kale, S. S., Mahajan, M., \& Smulay, S. (2016). Utility of sepsis screen in the early diagnosis of neonatal sepsis. Indian J Neonatal Med Res, 4(3), 1001-7.

Benitz, W. E., Han, M. Y., Madan, A., \& Ramachandra, P. (1998). Serial serum C-reactive protein levels in the diagnosis of neonatal infection. Pediatrics, 102(4), e41-e41.

Chirico, G., \& Loda, C. (2011). Laboratory aid to the diagnosis and therapy of infection in the neonate. Pediatric reports, 3(1), 1-5.

Chugh, K., Aggarwal, B. B., Kaul, V. K., \& Arya, S. C. (1988). Bacteriological profile of neonatal septicemia. The Indian Journal of Pediatrics, 55(6), 961-965.

Chiesa, C., Panero, A., Osborn, J. F., Simonetti, A. F., \& Pacifico, L. (2004). Diagnosis of neonatal sepsis: a clinical and laboratory challenge. Clinical chemistry, 50(2), 279287..

Celik, I. H., Demirel, F. G., Uras, N., Oguz, S. S., Erdeve, O., Biyikli, Z., \& Dilmen, U. (2010). What are the cut-off levels for IL-6 and CRP in neonatal sepsis?. Journal of clinical laboratory analysis, 24(6), 407-412.

Dulay, A. T., Buhimschi, I. A., Zhao, G., Bahtiyar, M. O., Thung, S. F., Cackovic, M., \& Buhimschi, C. S. (2015). Compartmentalization of acute phase reactants Interleukin6, C-Reactive Protein and Procalcitonin as biomarkers of intra-amniotic infection and chorioamnionitis. Cytokine, 76(2), 236-243.

Da Silva, O., Ohlsson, A., \& Kenyon, C. (1995). Accuracy of leukocyte indices and C-reactive protein for diagnosis of neonatal sepsis: a critical review. The Pediatric infectious disease journal, 14(5), 362-366.

Døllner, H., Vatten, L., \& Austgulen, R. (2001). Early diagnostic markers for neonatal sepsis: comparing C-reactive protein, interleukin-6, soluble tumour necrosis factor receptors and soluble adhesion molecules. Journal of clinical epidemiology, 54(12), 1251-1257.

Emami, S., Kalani, M., \& Mohaddes, G. A. (2016). Diagnostic role of serum haptoglobin level in early onset neonatal sepsis. Iranian Journal of Neonatology IJN, 7(2), 7-10.

El-Sonbaty MM, AlSharany W, Youness ER, Mohamed NA, Abdel-Hamid TA, Abdel-Razek A-RA. Diagnostic utility of biomarkers in diagnosis of early stages of neonatal sepsis in neonatal intensive care unit in Egypt. Egyptian Pediatric Association Gazette. 2016;64(2):91-6.

Gandhi, S., Ranjan, K. P., Ranjan, N., Sapre, N., \& Masani, M. (2013). Incidence of neonatal sepsis in tertiary care hospital: An overview. 
Hisamuddin, E., Hisam, A., Wahid, S., \& Raza, G. (2015). Validity of C-reactive protein (CRP) for diagnosis of neonatal sepsis. Pakistan journal of medical sciences, 31(3), 527.

Hofer, N., Müller, W., \& Resch, B. (2011). Non-infectious conditions and gestational age influence C-reactive protein values in newborns during the first 3 days of life. Clinical chemistry and laboratory medicine, 49(2), 297-302.

Hofer, N., Müller, W., \& Resch, B. (2013). The Role of C-reactive protein in the Diagnosis of Neonatal Sepsis, Neonatal Bacterial Infection, Prof. Bernhard Resch (Ed.).

Jeyaganguli, D., Velvizhi, G., Sucilathangam, G., \& Revathy, C. (2018). Diagnostic value of C-reactive protein and hematological markers in neonatal sepsis. Int J Curr Microbial APA Sci, 7(3), 722-7.

Kheir, A. E., Jobara, G. A., Elhag, K. M., \& Karar, M. Z. (2013). Qualitative C-reactive protein as a marker of neonatal sepsis in a tertiary neonatal unit in Sudan. Healthcare in Lowresource Settings, 1(2), e21-e21.

Kishk, R. M., Mandour, M. F., Farghaly, R. M., Ibrahim, A., \& Nemr, N. A. (2014). Pattern of blood stream infections within neonatal intensive care unit, Suez Canal University hospital, Ismailia, Egypt. International journal of microbiology, 2014.

Kayange, N., Kamugisha, E., Mwizamholya, D. L., Jeremiah, S., \& Mshana, S. E. (2010). Predictors of positive blood culture and deaths among neonates with suspected neonatal sepsis in a tertiary hospital, Mwanza-Tanzania. BMC pediatrics, 10(1), 1-9.

Labib, A. Z., Mahmoud, A. B., Eissa, N., El Gendy, F. M., Soliman, M. A., \& Aly, A. A. (2013). Early diagnosis of neonatal sepsis: a molecular approach and detection of diagnostic markers versus conventional blood culture. Int. J. Microbiol. Res, 4, 77-85.

Meem, M., Modak, J. K., Mortuza, R., Morshed, M., Islam, M. S., \& Saha, S. K. (2011). Biomarkers for diagnosis of neonatal infections: A systematic analysis of their potential as a point-of-care diagnostics. Journal of global health, 1(2), 201.

Magudumana, O., Ballot, D. E., Cooper, P. A., Trusler, J., Cory, B. J., Viljoen, E., \& Carter, A. C. (2000). Serial interleukin 6 measurements in the early diagnosis of neonatal sepsis. Journal of tropical pediatrics, 46(5), 267-271.

Mondal, G. P., Raghavan, M., Bhat, B. V., \& Srinivasan, S. (1991). Neonatal septicaemia among inborn and outborn babies in a referral hospital. The Indian Journal of Pediatrics, 58(4), 529-533.

Ngwa, D. N., \& Agrawal, A. (2019). Structure-function relationships of C-reactive protein in bacterial infection. Frontiers in immunology, 10, 166.

Noor, M. K., Shahidullah, M., Rahman, H., \& Mutanabbi, M. (2008). Interleukin-6: a sensitive parameter for the early detection of neonatal sepsis. Bangabandhu Sheikh Mujib Medical University Journal, 1(1), 1-5.

Polin, R. A. (2012). Management of neonates with suspected or proven early-onset bacterial sepsis. Pediatrics, 129(5), 1006-1015.

Poornima, M. (2017). Haematologial Scoring System: An Early Predictor of Neonatal Sepsis as Compared to Blood Culture (Doctoral dissertation, Coimbatore Medical College, Coimbatore). 
Parviz, A., Abolfazl, M., Mohammad Mahdi, D., Hassan, J., Neda, E., \& Negin, M. (2014). Comparison of serum IL-1beta and C-reactive protein levels in early diagnosis and management of neonatal sepsis. Le infezioni in medicina, (4), 296-301.

Shane, A. L., Sánchez, P. J., \& Stoll, B. J. (2017). Neonatal sepsis. The lancet, 390(10104), 1770-1780.

Sherwin, C., Broadbent, R., Young, S., Worth, J., McCaffrey, F., Medlicott, N. J., \& Reith, D. (2008). Utility of interleukin-12 and interleukin-10 in comparison with other cytokines and acute-phase reactants in the diagnosis of neonatal sepsis. American journal of perinatology, 25(10), 629-636.

Shah, B. A., \& Padbury, J. F. (2014). Neonatal sepsis: an old problem with new insights. Virulence, 5(1), 170-178.

Süner, A., Karaoğlan, I., METE, A. Ö., Namiduru, M., Boşnak, V., \& Baydar, I. (2015). Assessment of bloodstream infections and risk factors in an intensive care unit. Turkish journal of medical sciences, 45(6), 1243-1250.

Ucar, B., Yildiz, B., Aksit, M. A., Yarar, C., Colak, O., Akbay, Y., \& Colak, E. (2008). Serum Amyloid A, Procalcitonin, Tumor Necrosis Factor-, and Interleukin-1 Levels in Neonatal Late-Onset Sepsis. Mediators of inflammation, 2008.

Vinay, B. S., Girish, G. N., Adhikari, S., \& Hugara, S. (2015). Evaluation of septic screen as a diagnostic tool for neonatal sepsis in a tertiary hospital at Mysore. Sch J App Med Sci, 3(2G), 1005-1010. 


\section{الملخص العربى \\ علامات التشخيص المبكر للتسمم الوليدى}

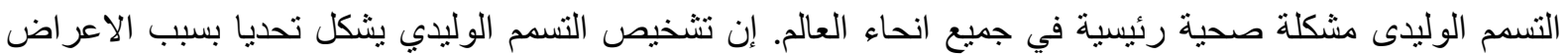

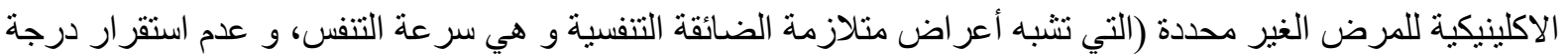

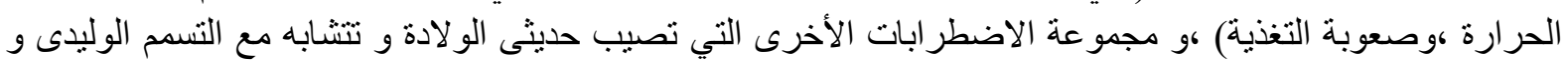

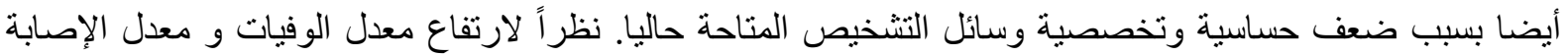

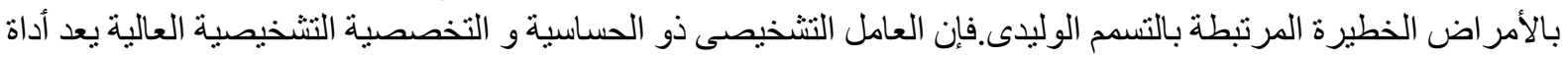

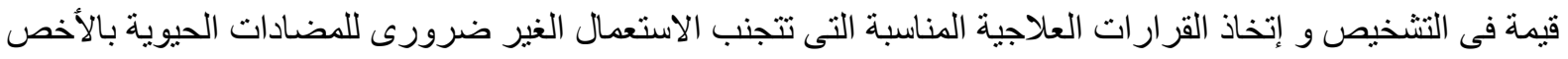

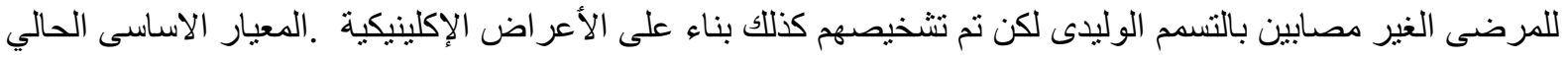

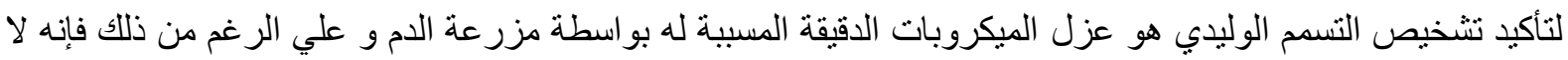

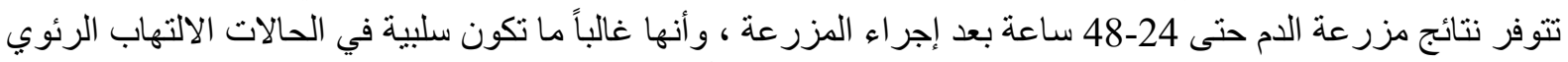

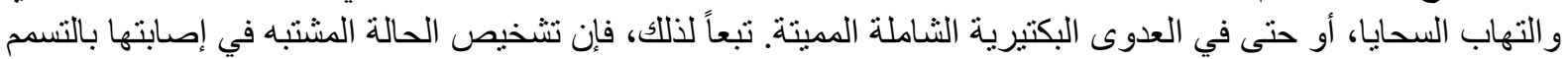

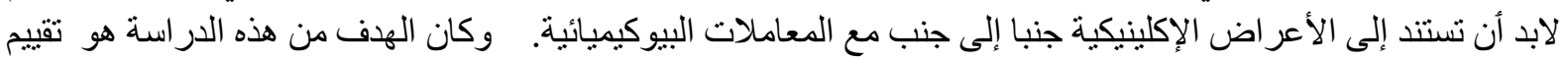

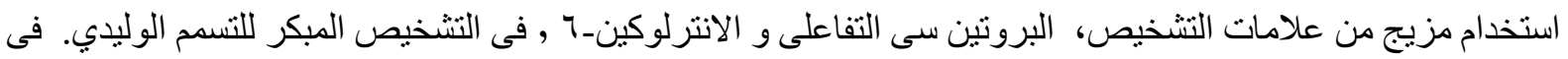

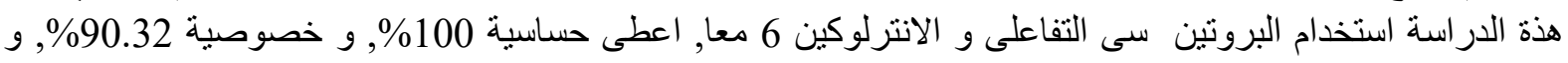

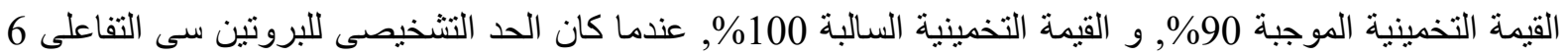

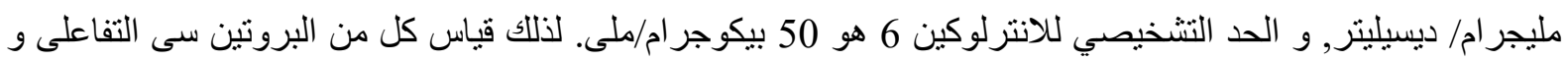

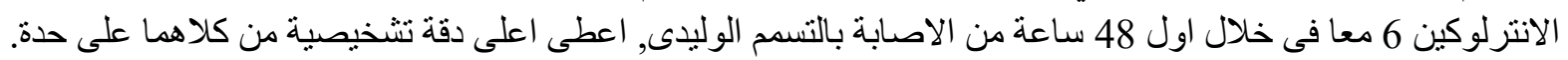

